\title{
Experimental Study of Multi-Magnet Excitation for Enhancing Micro-Power Generation in Piezoelectric Vibration Energy Harvester
}

\author{
Arūnas KLEIVA*, Rolanas DAUKŠEVIČIUS** \\ *Kaunas University of Technology, Studentu 56, 44025 Kaunas, Lithuania, E-mail: arunas.kleiva@ktu.edu \\ **Kaunas University of Technology, Studentu 56, 44025 Kaunas, Lithuania, E-mail: rolanas.dauksevicius@ktu.lt \\ crossref http://dx.doi.org/10.5755/j01.mech.25.3.22968
}

\section{Introduction}

Continuously increasing demand for autonomous self-charging devices requires effective implementation of energy harvesting methods in order to reduce heavy reliance on inconvenient batteries. The demand is fueled by progress in ultralow-power microelectronics and microfabrication techniques, which impel the development of portable and wearable smart devices for navigation, communication, fitness tracking, etc. [1-7].

Research work on vibration energy harvesting demonstrated that human-based mechanical energy is useable for conversion to electrical energy. It is mostly realized via piezoelectric, electrostatic, electromagnetic and triboelectric methods. This study is concerned with piezoelectric vibration energy harvesters (P-VEHs), which are commonly used due to sufficiently high energy density and relative ease of implementation at macro and micro scales [1-7]. However, it is very difficult to ensure stable high-power generation with wearable P-VEHs since human body parts move at very low frequencies (usually, up to several hertz) and high amplitudes, meanwhile piezoelectric transducers used in P-VEHs may deliver practically useable power levels only when excited to resonate at high frequencies (> 50-100 Hz), accompanied by very low vibration amplitudes [6-8]. This major mismatch between the excitation and operation conditions of the transducers is usually resolved by means of mechanical frequency upconversion (MFU) [9-13]. Effectively functioning MFU mechanism in wearable P-VEH must consistently provide sharp impulsive excitation (i.e. shock loading) of the transducer under varying low-frequency biomechanical excitations (e.g. limb movements) in order the transducer could engage in high-frequency free vibrations at its natural frequency [9-13]. MFU is realized by using either contactbased $[9,10]$ or contactless interaction [11-14]. The latter mostly relies on excitation via magnetic coupling and is better suited for wearable applications, which require noiseless operation. Moreover, due to absence of contactinduced wear of surfaces, it is easier to ensure long-term damage-free operation of P-VEHs that are based on magnetic excitation. Magnetic MFU is usually achieved by abruptly deflecting and releasing (i.e. plucking) transducer through the action of magnetic coupling forces that are induced between the moving excitation (driving) magnet and (ferro)magnetic tip mass (mounted on the transducer) [11-14]. Due to the inherently complicated nature of magnetic coupling (i.e. magnetic force impulses have relatively complex shapes [13]), it is very difficult to implement a compact and low-weight wearable P-VEH. More specifically, it is challenging to obtain stable impulsive magnetic plucking of the transducer by using excitation magnet that is set into motion only by inertial forces generated during relatively slow body movements.

This paper presents experimental demonstration of a more effective contactless MFU, which is based on multimagnet plucking. An experimental setup is implemented in order to investigate the process of multi-magnet plucking and establish the most favorable conditions that allow to enhance vibration energy harvesting performance when the transducer is magnetically excited by two or more moving magnets during an operation cycle (single plucking event). Although the reported results are targeted towards biomechanical energy harvesting, they are also relevant to other PVEH use cases that are characterized by low-frequency excitation conditions such as those encountered in automotive, civil engineering and other applications.

\section{Structure and operation of magnetically plucked pie- zoelectric vibration energy harvester}

Fig. 1 illustrates structure and operation of the investigated magnetically plucked $\mathrm{P}-\mathrm{VEH}$ including singlemagnet and multi-magnet excitation configurations. In order to measure generated voltage signals and derive average power output during different multi-magnet excitation conditions we used a cantilevered piezoceramic (PZT-5A) bimorph transducer made by Piezo Systems Inc. with the dimensions of $31.8 \times 57.2 \mathrm{~mm}^{2}$ (Table 1 ). This type of transducer was chosen due to relatively high piezoelectric coefficients, permittivity and high electrical outputs, which are useable for vibration energy harvesting purposes [15]. A neodymium cuboidal magnet with dimensions of $5 \times 5 \times 3 \mathrm{~mm}^{3}$ and magnetization N52 was attached at the free end of the cantilevered transducer. This magnet is referred to as the driven magnet. Identical magnets were attached on a rotating table with radius $r_{T}=115 \mathrm{~mm}$ (Fig. 2) in order to act as the driving magnets that are used to pluck the transducer. During table rotation the driving magnets move past the driven one, which leads to magnetic coupling and subsequent decoupling, thereby deflecting and releasing (plucking) the transducer, respectively. The width of zone where magnetic coupling is active is approximately 3 times larger than the width of the magnets, i.e. $15 \mathrm{~mm}$. It may be safely assumed that those $15 \mathrm{~mm}$ are travelled by the driving magnets in a linear trajectory since this distance is nearly 50 times smaller than the circumference of the table. Two different magnetic plucking configurations were investigated: i) in-plane (Fig. 1, b) and ii) out-of-plane (Fig. 1, c) by using 1,2 and 3 driving magnets. 


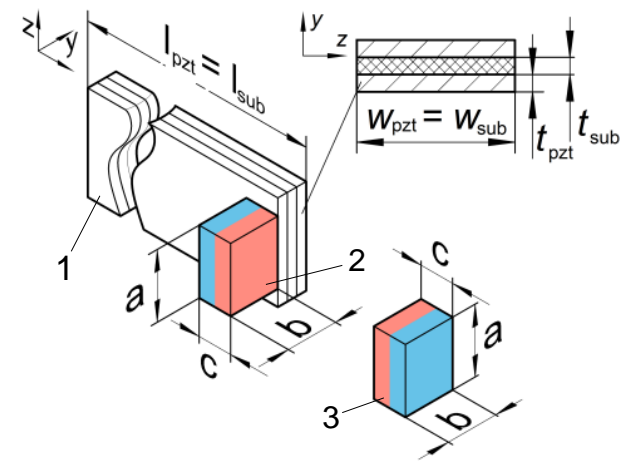

a

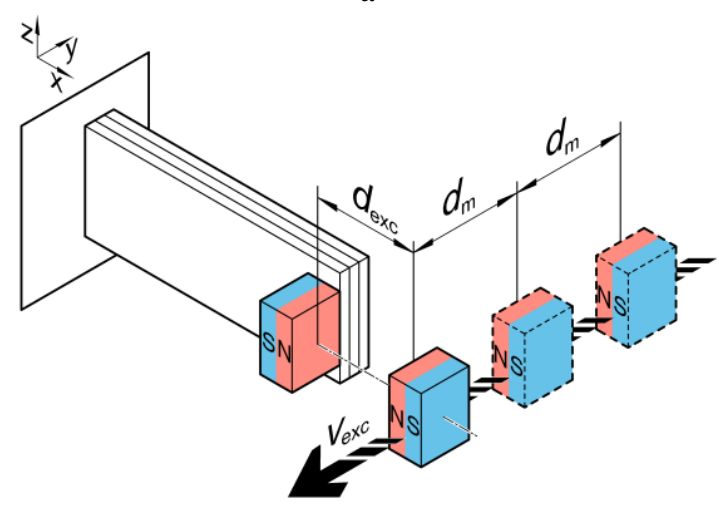

b

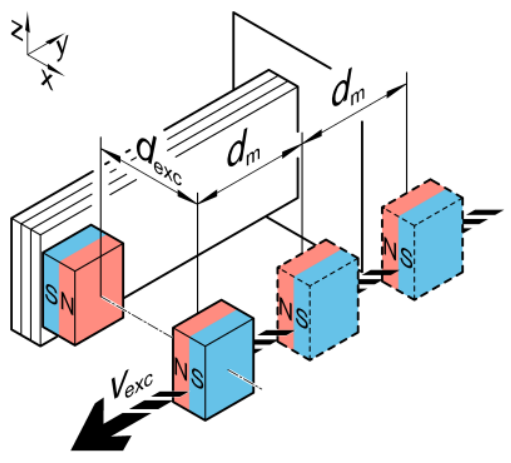

c

Fig. 1 Schematics of magnetically plucked transducer: a) geometric parameters of 1 - cantilevered bimorph, 2 - magnetic tip mass (driven magnet) and 3 - driving magnet, $l_{p z t}, w_{p z t}$ and $l_{s u b}, w_{s u b}-$ length and width of the piezoceramic and passive layers of the bimorph, respectively, $t_{p z t}$ - thickness of piezoceramic layers, $t_{s u b}$ - thickness of the passive layer (substrate), $a, b$ and $c$-height, thickness and width of the driving and driven magnets; b) In-plane and c) out-of-plane magnetic plucking configurations. $v_{e x c}-$ plucking speed, $d_{e x c}-$ gap between the driven and driving magnets, $d_{m}$ - gap between the driving magnets

The driving and driven magnets were oriented in a repulsive configuration. It is noted that the repulsive interaction is more favorable from viewpoint of practical implementation since there is no possibility that the driving and driven magnets will interlock during operation. Gap size $d_{e x c}$ $=7 \mathrm{~mm}$ between the magnets was chosen arbitrarily so that the transducer could be sufficiently deflected by the magnetic forces. For a given transducer, the dynamics of the magnetic plucking process and the generated electrical outputs are primarily governed by the motion speed of driving magnet (plucking speed) $v_{\text {exc }}$.
Table 1

Parameters of the investigated magnetically plucked piezoelectric vibration energy harvester

\begin{tabular}{|l|c|c|c|}
\hline Parameter & Unit & Transducer & Magnets \\
\hline Material & - & PZT-5A & NdFeB \\
\hline PZT-5A layers: & & & \\
length $\ell_{p z t}$ & $\mathrm{~mm}$ & 57.2 & - \\
width $w_{p z t}$ & $\mathrm{~mm}$ & 31.8 & - \\
thickness $t_{p z t}$ & $\mathrm{~mm}$ & 0.265 & - \\
\hline Passive (brass) layer: & & & \\
length $\ell_{s u b}$ & $\mathrm{~mm}$ & 57.2 & - \\
width $w_{\text {sub }}$ & $\mathrm{mm}$ & 31.8 & - \\
thickness $t_{\text {sub }}$ & $\mathrm{mm}$ & 0.14 & - \\
\hline Coupling coefficient: & & & \\
$k_{31}$ & - & 0.35 & - \\
$k_{33}$ & - & 0.72 & - \\
\hline Driven magnet: & & & \\
length $a$ & $\mathrm{~mm}$ & - & 5 \\
width $b$ & $\mathrm{~mm}$ & - & 5 \\
thickness $c$ & $\mathrm{~mm}$ & - & 3 \\
mass $m_{T M}$ & $\mathrm{~g}$ & - & 0.57 \\
magnetization & - & - & $\mathrm{N} 52$ \\
residual magnetism $B_{r}$ & $\mathrm{~T}$ & - & 1.45 \\
\hline Driving magnet: & & & \\
length $a$ & $\mathrm{~mm}$ & - & 5 \\
width $b$ & $\mathrm{~mm}$ & - & 5 \\
thickness $c$ & $\mathrm{~mm}$ & - & 3 \\
mass $m_{D M}$ & $\mathrm{~g}$ & - & 0.57 \\
magnetization & - & - & $\mathrm{N} 52$ \\
residual magnetism $B_{r}$ & $\mathrm{~T}$ & - & 1.45 \\
\hline Young's modulus: & & & \\
$E_{\text {sub }}$ & $\mathrm{GPa}$ & 120 & \\
$E_{T M}=E_{D M}$ & $\mathrm{GPa}$ & & 160 \\
\hline Fundamental frequency $f_{0}$ & & & \\
(without tip mass) & $\mathrm{Hz}$ & 110 & - \\
\hline Capacitance $C_{P T}$ & $\mathrm{nF}$ & 200 & - \\
\hline Mechanical quality factor $Q_{m}$ & & & \\
(damping ratio), measured & - & $33(0.015)$ & - \\
with affixed tip mass & & & \\
\hline
\end{tabular}

The highest output is generated when $v_{\text {exc }}$ is sufficiently high to induce true impulsive excitation that leads to transient resonance, which is characterized by the highest amplitudes of the induced dynamic and electric transient responses [16]. In the investigated multi-magnet plucking case, the distance between the driving magnets $d_{m}=20 \mathrm{~mm}$ (Fig. 1, b) determines the impulse train period $t_{m}=d_{m} / v_{\text {exc }}$ (Fig. 3, a).

\section{Experimental setup}

Magnetically plucked P-VEH was tested by measuring electric and magnetic transient responses in experimental setup shown in Fig. 2. The driving magnets were attached on a custom-made rotating table $\left(r_{T}=115 \mathrm{~mm}\right)$, which was mounted on a DC motor. The rotational speed $\omega_{\text {rpm }}$ was adjusted in the range of $31-123 \mathrm{rpm}$, which corresponds to the plucking speed of $v_{e x c}=0.75-2.95 \mathrm{~m} / \mathrm{s}$ $\left(v_{e x c}=r_{T} \omega_{r p m}(2 \pi / 60)\right)$. The driven magnet was tightly fastened on the free end of the transducer, which was displaced $d_{\text {exc }}=7 \mathrm{~mm}$ away from the rotating table.

The measurement subsystem includes several instruments (Fig. 2).

$\omega_{r p m}$ was recorded using Polytec rotational laser Doppler vibrometer consisting of sensor head RLV-500 and controller RLV-5000. Magnetic flux densities along $x$-axis 
$\left(B_{x}\right)$ and $y$-axis $\left(B_{y}\right)$ were recorded simultaneously with two magnetometer Hall probes, which were placed in the middle between the driving and driven magnets. Digital oscilloscope PICO 6403 with software PicoScope 6 were used to capture and process signals of the magnetometers and the transducer, which was connected to the matched load resistor $R_{M L}$. All the measurements were made with P-VEH operating in the impulsive excitation regime, which means that the total transient response is largely dominated (energywise) by free vibrations characterized by a single (natural) frequency $f_{n}=80 \mathrm{~Hz}$ (more than $90 \%$ of electrical energy is generated during free vibrations under impulsive excitation conditions [16]). Therefore, value of matched load resistance may theoretically estimate as $R_{M L}=1 / 2 \pi f_{n} C_{P T}=$ $=9.95 \mathrm{k} \Omega\left(C_{P T}-\right.$ capacitance of the transducer $)$. This resistance value may be considered as near-optimal one since in this case $\mathrm{P}-\mathrm{VEH}$ generates (near) maximal power output under impulsive plucking at $v_{\text {exc }}=0.75-2.95 \mathrm{~m} / \mathrm{s}$.

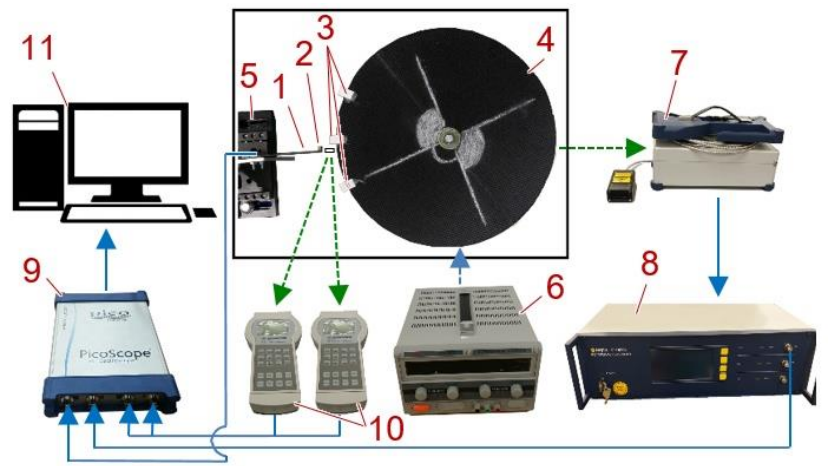

Fig. 2 Principal scheme of experimental setup used for electrical characterization of the P-VEH: 1 - transducer (Piezo Systems T226-A4-503Y), 2 - driven magnet, 3 - driving magnet(s) in cartridge(s); 4 - rotating table, 5 - micropositioning stage, 6 - DC power supply (Mastech HY3020E) for DC motor, 7 - Rotational LDV sensor head (Polytec RLV-500), 8 - LDV controllers (RLV-5000), 9 - digital oscilloscope (PICO 6403), 10 - Magnetometers (Magnet-Physik FH-54) with sensor (transverse Hall probe HS-TGB5), 11 data processing (computer)

For establishing the relationship between $v_{e x c}$ and energy harvesting performance we analyzed temporal excitation parameters that characterize the sharpness of the magnetic impulses and govern the dynamics of the excitation process (Fig. 3, a): $B_{x}$ impulse duration $t_{M P}\left(B_{x}\right)$ and duration of $B_{y}$ signal direction reversal $t_{j u m p}\left(B_{y}\right)$. Accordingly, by taking half of these parameters, we obtain impulse rise times, which are called here as ramping times $t_{\text {ramp }}\left(B_{x}\right)$ and $t_{\text {ramp }}\left(B_{y}\right)$. For clarity, ramping time refers to the excitation process (impulse), meanwhile the rise time refers to the response of the transducer and in the case of underdamped dynamic system may be defined as $t_{\text {rise }}=0.25 T_{n}=3.125 \mathrm{~ms}$ $\left(T_{n}=1 / f_{n}\right)$. Measurement results in Fig. 3, b show that $t_{\text {ramp }}\left(B_{x}\right)$ and $t_{\text {ramp }}\left(B_{y}\right)$ values are approximately equal (the difference is less than $5 \%$ ). We observe that with increasing $v_{\text {exc }}$ both ramping times diminish nonlinearly.

Impulse train period $t_{m}$ also follows nonlinear variation trend with changing $v_{\text {exc }}$. In terms of magnetic field measurements, it should be pointed out that unavoidably the absolute magnitudes of $B_{x}$ and $B_{y}$ signals are not fully accurate because the driving magnets were moving, while the magnetometer was kept stationary. This explains the fluctuations of magnetic signals after the decoupling (Fig. 5, a). Moreover, the magnetometers registered magnetic field in the middle of the gap between the magnets, while magnetic forces that deflect the transducer, act directly on the driven magnet. Our previous study demonstrated that the measured $t_{\text {ramp }}\left(B_{x}\right)$ and $t_{\text {ramp }}\left(B_{y}\right)$ values are higher approximately by $10 \%$ with respect to the actual magnetic forces [16]. However, it is noted that these quantitative discrepancies do not have consequential effect on the results of this research work, particularly in terms of relationships between governing temporal parameters (ramping times, rise times, periods).

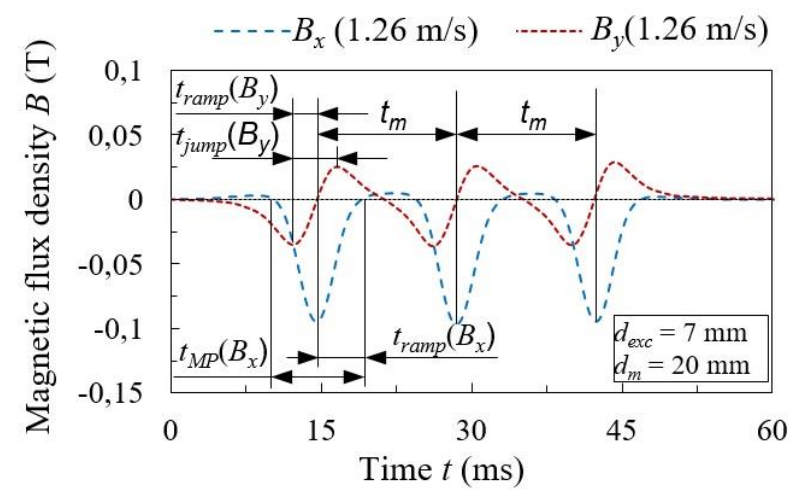

a

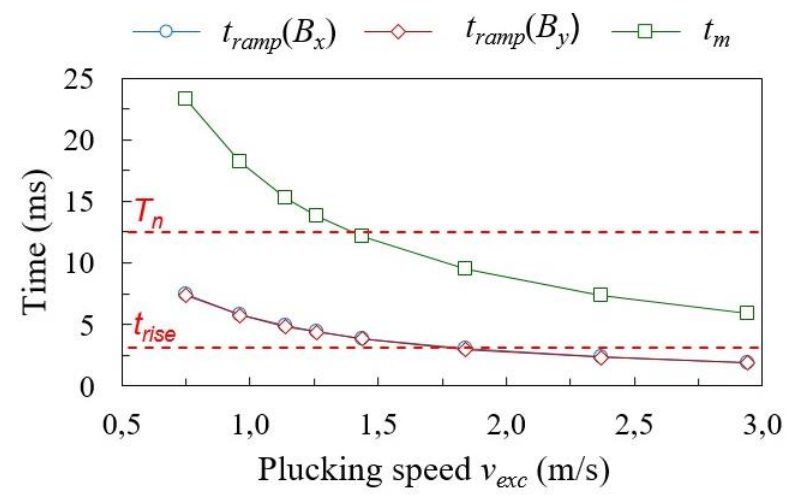

b

Fig. 3 a) Measured impulses of axial $B_{x}$ and transverse $B_{y}$ magnetic fields at $v_{e x c}=1.26 \mathrm{~m} / \mathrm{s}$; b) Temporal excitation parameters as a function of $v_{\text {exc }}\left(d_{\text {exc }}=7 \mathrm{~mm}\right)$

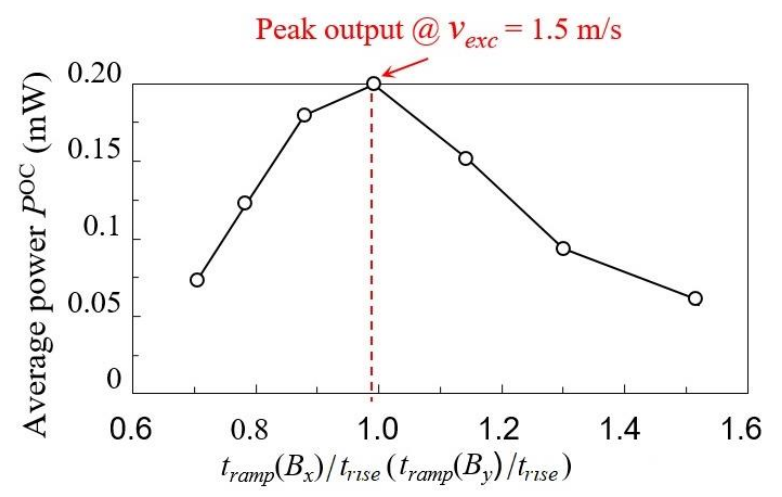

Fig. 4 Measured average power $P^{O C}$ as a function of dimensionless ramping times $\left(d_{e x c}=7 \mathrm{~mm}\right)$. 


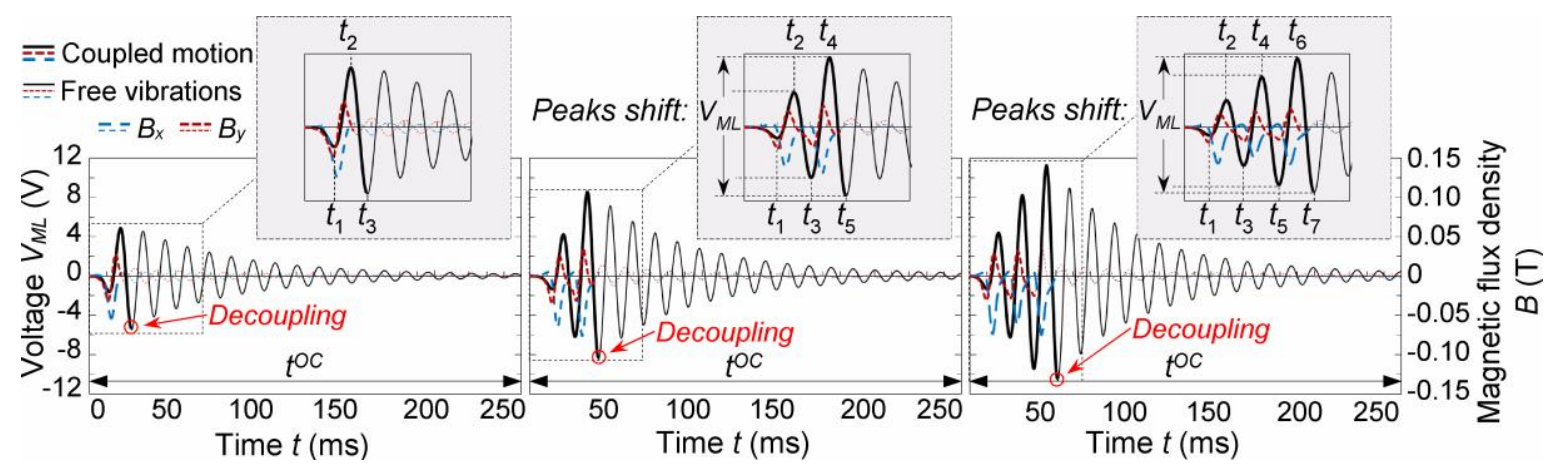

$\mathrm{b}$

c

Fig. 5 Measured transient signals of generated voltage $V_{\mathrm{ML}}$ and magnetic flux density $B_{x}$ and $B_{y}$ when P-VEH operates in (a) single-, (b) double- and (c) triple-magnet in-plane plucking regime $\left(d_{e x c}=7 \mathrm{~mm}, d_{m}=20 \mathrm{~mm}, v_{e x c}=1.5 \mathrm{~m} / \mathrm{s}\right)$.

\section{Experimental analysis}

In order to identify excitation conditions for inducing transient resonance (i.e. for generating maximized power output), we measured and analyzed a series of transient responses of voltage, average power and magnetic flux density (Fig. 4) by varying $v_{\text {exc }}$ in the range of $0.75-2.95$ $\mathrm{m} / \mathrm{s}$, which corresponds to ratios of $t_{\text {ramp }}\left(B_{x}\right) / t_{\text {rise }}=t_{\text {ramp }}\left(B_{y}\right)$ $/ t_{\text {rise }}=0.7-1.5$ (Fig. 4, a). Total transient response of duration $t^{O C}=250 \mathrm{~ms}$ was used for calculating $V_{r m s}$ and $P_{a v}$ as follows [17]:

$$
P_{a v}=\frac{V_{r m s}^{2}}{R_{L}}=\frac{1}{R_{L}}\left(\sqrt{\frac{1}{k}\left(\sum_{i=1}^{k} V^{2}\left(t_{i}\right)\right)}\right)^{2},
$$

here: $V\left(t_{i}\right)$ is instantaneous voltage values on the resistor $R_{M L}=9.95 \mathrm{k} \Omega, k$ is quantity of analyzed $V\left(t_{i}\right)$ values.

Average power that is generated during the complete operational cycle is denoted as $P^{O C}$. Upon plucking the induced transient response begins with the forced vibration stage (coupled motion), which continues until time instance $t_{3}$ (Fig. 5, a). In the analyzed impulsive plucking case, after decoupling the response continues with free vibrations, which continues until nearly full decay at $t^{O C}=250 \mathrm{~ms}$.

Dynamic characteristics of the free vibration response are predetermined by the dynamic characteristics at the end of the forced vibration stage. Measurement results in Fig. 4 demonstrate that P-VEH delivers peak power at $v_{\text {exc }}=1.5 \mathrm{~m} / \mathrm{s}\left(t_{\text {ramp }}\left(B_{x}\right) / t_{\text {rise }}=t_{\text {ramp }}\left(B_{y}\right) / t_{\text {rise }}=0.94\right)$. It is observed that the average power starts to diminish when plucking speed shifts away from the resonant plucking speed $v_{\text {exc }}=1.5 \mathrm{~m} / \mathrm{s}$ because acting excitation forces ramp up either insufficiently rapidly or overly rapidly with respect to the natural responsiveness of the transducer (defined by its rise time $t_{\text {rise }}=0.25 T_{n}$ ).

In general, dominant deflection forces in the $\mathrm{P}$ VEH are different for the in-plane (Fig. 1, b) and out-ofplane (Fig. 1, c) plucking configurations. During the inplane plucking, as the driving magnet comes near the driven one, repulsive transverse force (represented by the magnetic field component $B_{y}$ ) starts to deflect the transducer from its equilibrium position towards the approaching magnet (Fig. 5, a). Subsequently, the driving magnet deflects transducer until a limit point $t_{1}$. As the magnet moves onwards and passes the equilibrium position, the direction of $B_{y}$ reverses, which results in transducer deflecting to the opposite side of the equilibrium position, where another limit point $t_{2}$ is reached. Closer inspection of Fig. 5, a reveals that $B_{x}$ and $B_{y}$ do not abruptly vanish at $t_{2}$, which indicates that magnetic coupling vanishes in a relatively gradual manner.

Magnetic decoupling process occurring during $B_{y}$ deactivation has an influence on the motion of the transducer (at least) until $t_{3}$. In the case of the impulsive excitation, it is reasonable to assume that $t_{3}$ represents a complete decoupling, which corresponds to the release of the transducer, thereby denoting the end of the forced vibrations (coupled motion). When analyzing electrical responses of the multi-magnet P-VEH operating in the in-plane plucking regime with either two (Fig. 5, b) or three (Fig. 5, c) driving magnets, we observe generation of several contiguous magnetic $B_{x}$ and $B_{y}$ impulses (periodic impulse train), with the coupled motion stages ending at $t_{5}$ and $t_{7}$, respectively.

Meanwhile, transducer deflections during the outof-plane plucking are caused by the axial magnetic repulsive force (represented by the magnetic field $B_{x}$ ), which is produced when the driving magnet approaches the driven one. Contrary to the in-plane plucking configuration, in this case, firstly, the driving magnet attracts the transducer until a limit point $t_{1}$ and then repels it to the opposite side of the equilibrium position $t_{2}$, followed by the decoupling (transducer release) and free vibrations. Analogously to the previous in-plane plucking case, we consider that time instances $t_{3}, t_{5}$ and $t_{7}$ represent the end of the coupled motion stage in the single-, double- and triple-magnet out-of-plane plucking regime, respectively (Fig. 6, a). We note that for the sake of brevity, Fig. 6 is used to visualize the electrical signals measured only during the triple-magnet plucking regime (impulsive excitation). Fig. 6, b serves to illustrate the resulting energy harvesting performance by providing transient signals of generated average power and total cumulative energy (derived from the $V_{M L}$ signal in Fig. 6, a).

Next, we compare P-VEH performance obtained during single- and multi-magnet plucking regimes. Fig. 7 shows that peak electrical outputs generated during singlemagnet in-plane plucking at the resonant plucking speed of $1.5 \mathrm{~m} / \mathrm{s}$ are $P^{O C}=0.190 \mathrm{~mW}$ and $0.042 \mathrm{~mJ}$. Double-magnet plucking (i.e. two driving magnets) allows to increase average power and total energy 3.2 times with respect to the single-magnet case. The addition of the third driving magnet during the triple-magnet plucking further increases the power output (5.9 and 1.9 times in comparison to the singleand double-plucking cases, respectively). Similar gains in vibration energy harvesting performance are also obtained in the out-of-plane plucking regime. 


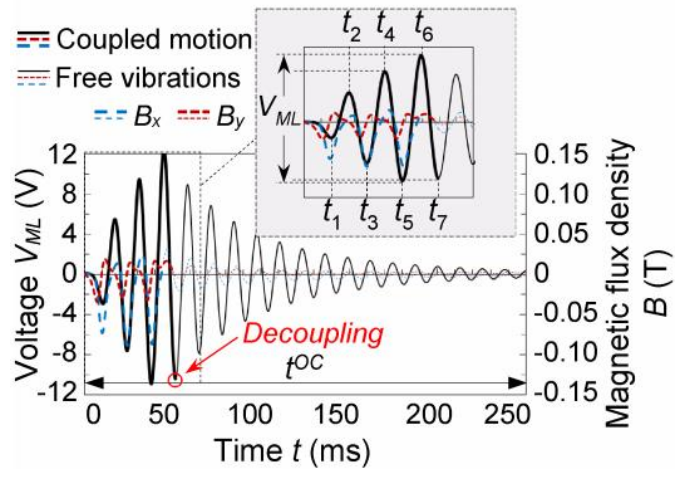

a

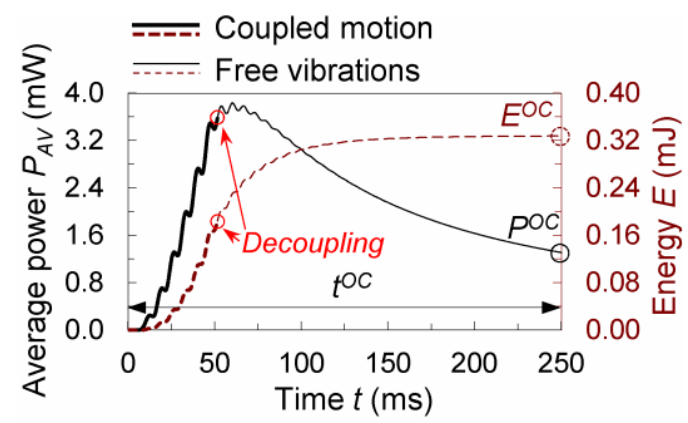

b

Fig. 6 a) Measured transient signals of generated voltage $V_{M L}$ and magnetic flux density $B_{x}$ and $B_{y}$; b) Calculated average power and total cumulative energy when P-VEH operates in the triple-magnet out-ofplane excitation regime $\left(d_{e x c}=7 \mathrm{~mm}, d_{m}=20 \mathrm{~mm}\right.$, $v_{\text {exc }}=1.5 \mathrm{~m} / \mathrm{s}$ )

It is observed that the average power increases 3.6 times when adding the second driving magnet and increases further 1.8 times when operating in the triple-magnet plucking regime. We note that during the triple-magnet out-ofplane plucking the P-VEH generates 6.7 times more average power in comparison to the conventional single-magnet operating regime. This gain in performance is even more substantial than in the case of the in-plane plucking. Overall, these experimental results demonstrate that it is possible to significantly boost power and energy outputs of the magnetically plucked P-VEH by employing consecutive impulsive excitation events (periodic impulse train) that induce resonant transient responses. Specifically, multi-magnet plucking regime may provide enhanced energy harvesting performance only when P-VEH is impulsively excited to engage in transient resonance. It implies that each consecutive excitation impulse must ensure that the ramping time of the dominant deflecting force is comparable to the transducer rise time $t_{\text {rise }}$ [16]. The largest gain in electrical outputs during the multi-magnet plucking regime is obtained when the ramping times are slightly smaller than the transducer rise time, i.e. when $t_{\text {ramp }}\left(B_{x}\right) / t_{\text {rise }} \cong t_{\text {ramp }}\left(B_{y}\right) / t_{\text {rise }} \cong 0.94$ the PVEH operates in transient resonance, which in the tested setup is obtained at $v_{\text {exc }} \cong 1.5 \mathrm{~m} / \mathrm{s}$.

Although not shown graphically, we note that electrical outputs drastically diminish when the system deviates from the transient resonance, i.e. when $v_{\text {exc }}$ shifts from the resonant plucking speed of $1.5 \mathrm{~m} / \mathrm{s}$. In such case the consecutive excitations due to the second or third driving magnets suppress transient response that has been initially induced by the first driving magnet.

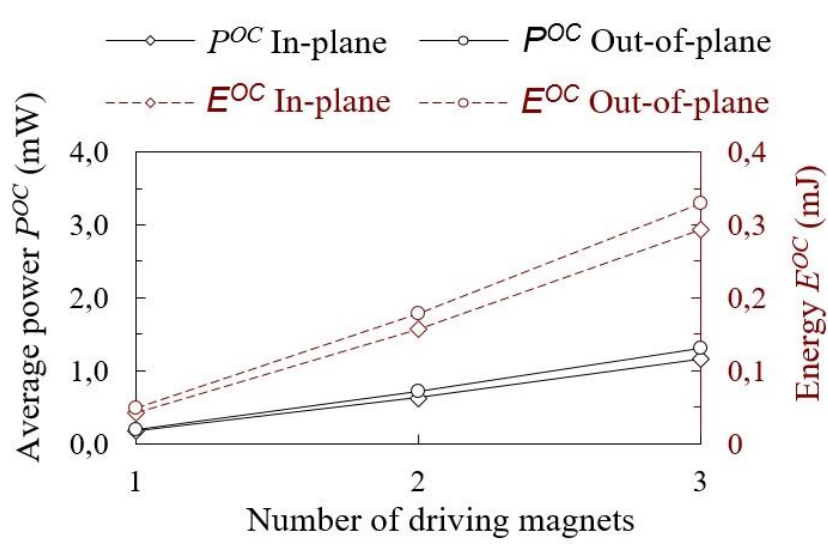

Fig. 7 Measured average power and total cumulative energy of the single-, double- and triple-magnet P-VEHs operating in the in-plane and out-of-plane plucking regimes at transient resonance $\left(d_{\text {exc }}=7 \mathrm{~mm}, d_{m}=20\right.$ $\mathrm{mm}, v_{\text {exc }}=1.5 \mathrm{~m} / \mathrm{s}$ )

In order to achieve gain in $\mathrm{P}-\mathrm{VEH}$ performance during multi-magnet plucking regime, firstly, it is necessary to identify the resonant plucking speed and then properly adjust the distance $d_{m}$ (and $t_{m}=d_{m} / v_{\text {exc }}$ accordingly) between the driving magnets. Fig. 3, b indicates that the point on $v_{\text {exc }}$ axis where experimental curve of $t_{m}$ intersects with the line of $T_{n}$ corresponds to the vicinity of transient resonance, i.e. peak power point at $v_{\text {exc }}=1.5 \mathrm{~m} / \mathrm{s}\left(t_{\text {ramp }}\left(B_{x}\right) / t_{\text {rise }}\right.$ $\left.=t_{\text {ramp }}\left(B_{y}\right) / t_{\text {rise }}=0.94\right)$ in Fig. 4). For example, in the case of the tested P-VEH setup, the resonant plucking speed $v_{\text {exc }}$ $=1.5 \mathrm{~m} / \mathrm{s}$ and the gap between driving magnets $d_{m}=20 \mathrm{~mm}$ ensure the required impulse train period $t_{m}$, i.e. they ensure that the conditions of $t_{m} / T_{n}=1$ and $t_{\text {ramp }}\left(B_{x}\right) / t_{\text {rise }} \cong t_{\text {ramp }}\left(B_{y}\right)$ $/ t_{\text {rise }} \cong 0.94$ are simultaneously satisfied. Thus, it may be generalized that the multi-magnet plucking may be beneficially exploited for power output increase only when the resonant plucking speed $v_{\text {exc }}$ and the gap between the driving magnets $d_{m}$ are tuned simultaneously in order to ensure that dimensionless ramping times $t_{\text {ramp }}\left(B_{x}\right) / t_{\text {rise }}, t_{\text {ramp }}\left(B_{y}\right) / t_{\text {rise }}$ and the dimensionless impulse train period $t_{m} / T_{n}$ are close to unity.

\section{Conclusions}

This paper presented experimental investigation of the method of more effective contactless mechanical frequency up-conversion, which relies on multi-magnet plucking of a piezoelectric vibration energy harvester having magnetic tip mass mounted at the free end of the cantilevered bimorph transducer. The proposed method exploits several consecutive magnetic impulsive excitations (periodic impulse train) acting during single plucking event in order to increase micro-power generation performance. It was established that there are two prerequisites for the method to be effective. First, the transducer must be impulsively excited via magnetic coupling forces and produce resonant transient responses leading to maximal power and energy outputs. Measurements indicate that P-VEH delivers peak outputs when the speed of the driving magnet $v_{\text {exc }}$ is high enough to produce magnetic field impulses that meet the criterion of $t_{\text {ramp }}\left(B_{x}\right) \cong t_{\text {rise }}$ for the out-of-plane plucking and $t_{\text {ramp }}\left(B_{y}\right) \cong t_{\text {rise }}-$ for the in-plane one. Second, it is necessary to select such separation between the driving magnets $d_{m}$ that allows the impulse train period to meet the criterion 
of $t_{m}=T_{n}$ as closely as possible. It was experimentally demonstrated that, in comparison to the conventional singlemagnet plucking regime, the proposed multi-magnet excitation method enables increase of the average power and total generated energy 3.2 or 5.9 times in the case of double- or triple-magnet in-plane plucking and 3.6 or 6.7 times - in the case of the out-of-plane plucking, respectively.

\section{Acknowledgments}

This research was funded by a grant No. S-MIP17-89 from the Research Council of Lithuania (project acronym: FLEXYMECH-3DP).

\section{References}

1. Briand, D.; Yeatman, E.; Roundy, S. 2015. Micro Energy Harvesting, Book Series:Advanced Micro and Nanosystems. https://doi.org/10.1002/9783527672943.ch10.

2. Dagdeviren, C.; Li, Z.; Wang, Z.L. 2017. Energy harvesting from the animal/human body for self-powered electronics, Annu. Rev. Biomed. Eng. 19: 85-108. https://doi.org/10.1146/annurev-bioeng-071516044517.

3. Choi, Y.M.; Lee, M.G; Jeon, Y. 2017. Wearable biomechanical energy harvesting technologies, Energies 10: 1483.

https://doi.org/10.3390/en10101483.

4. Romero, E. 2013. Powering Biomedical Devices, Elsevier Inc. https://doi.org/10.1016/C2012-0-06126-1.

5. Todaro, M. T.; Guido, F.; Mastronardi, V.; Desmaele, D.; Epifani, G.; Algieri, L; De Vittorio, M. 2017. Piezoelectric MEMS vibrational energy harvesters: Advances and outlook, Microelectron. Eng. 183184: 23-36. https://doi.org/10.1016/j.mee.2017.10.005.

6. Wei, C; Jing, X. 2017. A comprehensive review on vibration energy harvesting: Modelling and realization, Renewable Sustainable Energy Rev. 74: 1-18. https://doi.org/10.1016/j.mee.2017.10.005.

7. Priya, S; Inman, J.D. (eds) 2009. Energy Harvesting Technologies (New York: Springer). https://doi.org/10.1007/978-0-387-76464-1.

8. Yang, Z.; Erturk, A.; Zu, J. 2017. On the efficiency of piezoelectric energy harvesters, Extreme Mechanics Letters 15: 26-37. https://doi.org/10.1016/j.eml.2017.05.002.

9. Halim, M.A.; Cho, H; Park, J.Y. 2015. Design and experiment of a human-limb driven, frequency up-converted electromagnetic energy harvester, Energy Convers. Manage. 106: 393-404. https://doi.org/10.1016/j.enconman.2015.09.065.

10. Alghisi, D.; Dalola, S.; Ferrari, M.; Ferrari, V. 2015. Triaxial ball-impact piezoelectric converter for autonomous sensors exploiting energy harvesting from vibrations and human motion, Sens. Actuator A-Phys. 233: 569-581. https://doi.org/10.1016/j.sna.2015.07.020.

11. Pillatsch, P.; Yeatman, E. M.; Hssolmes, A. S. 2014. Magnetic plucking of piezoelectric beams for frequency up-converting energy harvesters, Smart Mater. Struct. 23: 025009 . https://doi.org/10.1088/0964-1726/23/2/025009.

12. Pozzi, M. 2016. Magnetic plucking of piezoelectric bimorphs for wearable energy harvester, Smart Mater. Struct. 25: 045008. https://doi.org/10.1088/0964-1726/25/4/045008.

13. Xue, T.; Roundy, S. 2017. On magnetic plucking configurations for frequency up-converting mechanical energy harvesters, Sensors Actuators A-Phys. 253: 101-11. https://doi.org/10.1016/j.sna.2016.11.030.

14. Abdul, H.; Miah, R.; Rantz, T.; Xue, S.; Roundy, S. 2018. Architectures for wrist-worn energy harvesting, Smart Materials and Structures 27(4):044001. https://doi.org/10.1088/1361-665X/aa94d6.

15. Piezo Material. Link via internet. View on 2019.02.20. https://piezo.com/pages/piezo-material.

16. Dauksevicius, R.; Kleiva, A.; Grigaliunas, V. 2018. Analysis of magnetic plucking dynamics in a frequency up-converting piezoelectric energy harvester, Smart Materials and Structures 27(8).

https://doi.org/10.1088/1361-665X/aac8ad.

17. Daintith, J. 2009. A Dictionary of Physics, https://doi.org/10.1093/acref/9780199233991.001.000.

\section{A. Kleiva, R. Dauksevicius}

\section{EXPERIMENTAL STUDY OF MULTI-MAGNET EXCITATION FOR ENHANCING MICRO-POWER GENERATION IN PIEZOELECTRIC VIBRATION ENERGY HARVESTER}

S u m m a r y

The reported work experimentally investigates a method of more effective contactless mechanical frequency up-conversion that is based on multi-magnet plucking of a piezoelectric vibration energy harvester. Several moving excitation magnets are used to produce a periodic impulse train, which during a single plucking event consecutively deflects and then releases the cantilevered transducer to freely oscillate, thereby enabling enhanced micro-power generation performance. It was established that the proposed method is effective if a couple conditions are met. First, the transducer must be impulsively excited to produce resonant transient responses, which occurs when the ramping time of the magnetic impulse is close to the transducer rise time (defined as a quarter of the natural period). Second, the gap between the moving excitation magnets must be tuned to ensure that the impulse train period is as close to the natural period as possible. Measurements indicate that, in comparison to the conventional single-magnet plucking case, the consecutive excitation with three moving magnets leads to nearly sixfold (seven-fold) increase in average power output and total generated energy during the in-plane (out-of-plane) plucking regime.

Keywords: vibration energy harvesting, piezoceramic transducer, frequency up-conversion, magnetic plucking, resonant transient responses.

Received March 15, 2019

Accepted June 14, 2019 